\title{
Quality control of malaria microscopy reveals misdiagnosed non-falciparum species and other microscopically detectable pathogens in Senegal
}

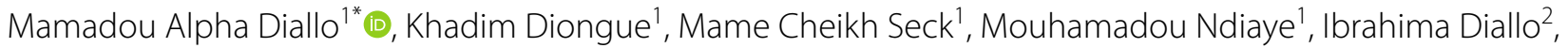 \\ Younouss Diedhiou' ${ }^{1}$ Tolla Ndiaye ${ }^{1}$, Yaye Die Ndiaye ${ }^{1}$, Aida Sadikh Badiane ${ }^{1}$ and Daouda Ndiaye ${ }^{1}$
}

\begin{abstract}
Background: In developing countries, malaria diagnosis relies on microscopy and rapid diagnostic tests. In Senegal, national malaria control program (NMCP) regularly conducts supervisory visits in health services where malaria microscopy is performed. In this study, expert microscopists assessed the performance of laboratory technicians in malaria microscopy.

Methods: The present external quality assessment (EQA) was conducted in three different areas of malaria transmission. Participants were laboratory technicians previously trained by NMCP on malaria microscopy. Stored read slides were randomly collected for blinded re-checking by expert microscopists. At the same time a set of 8 slides (3 positive P. falciparum and 5 negative slides) were submitted to participants for proficiency testing. Microscopists performance were evaluated on the basis of the errors rates on slide reading — high false positive (HFP), high false negative (HFN), low false positive (LFP) and low false negative (LFN) - and the calculation of their sensitivities and specificities relative to expert microscopy. Data were entered and analysed using Microsoft Excel software.
\end{abstract}

Results: A total of 450 stored slides were collected from 17 laboratories for re-checking. Eight laboratories scored $100 \%$ of correct reading. Only one major error was recorded (HFP). Six laboratories recorded LFN results: Borrelia, $P$. ovale, and low parasite densities (95 and $155 \mathrm{p} / \mu \mathrm{ll}$ ) were missed. Two $P$. falciparum slides were misidentified as $P$. malariae and one $P$. ovale slide as $P$. vivax. The overall sensitivities and specificities for all participants against expert microscopists were 97.8 and $98.2 \%$ respectively; Sensitivities and specificities of hospital microscopists (96.7 and 98.9\%) were statistically similar to those of health centre microscopists (98.5 and $97.8 \%$ respectively) $(p=0.3993$ and $\mathrm{p}=0.9412$ respectively). Overall, a very good agreement was noted with kappa value of $0.96\left(\mathrm{Cl}_{95 \%} 93.4-98.6 \%\right)$ relative to expert microscopy. Proficiency testing showed that among the 17 participants, 11 laboratories scored 100\% of correct reading. Three LFN and four LFP results were recorded respectively. The $P$. falciparum slide with Maurer dots was misidentified as P. ovale in 1 centre and the same slide was misread as P. vivax in another centre; No major error (HFP or HFN) was noted.

Conclusion: EQA of malaria microscopy showed an overall good performance especially regarding P. falciparum detection. However, efforts need to be made addressing the ability to detect non-falciparum species and others endemic blood pathogens such as Borrelia. The further NMCP training sessions and evaluations should consider those aspects to expect high quality-assured capacity for malaria microscopy.

\footnotetext{
*Correspondence: mamadoualpha.diallo@ucad.edu.sn

1 Laboratory of Parasitology and Mycology, Cheikh Anta Diop University,

Avenue Cheikh Anta Diop, BP 5005 Fann, Dakar, Senegal

Full list of author information is available at the end of the article
} 
Keywords: External quality assessment, EQA, Microscopy, Diagnosis, Malaria, Senegal

\section{Background}

Despite the development of new diagnostic tools, microscopy still remains the gold standard method for malaria diagnosis [1]. Microscopy of thick and thin smears is relatively simple and inexpensive, and particularly suitable to rapidly diagnose malaria. Moreover, microscopy allows species identification and parasite count [2], and it is also suitable to diagnose infections with other pathogens that may be detected microscopically in routinely Giemsa-stained smears, such as spirochetes of Borrelia, which are common in Senegal [3]. However, reliability of malaria microscopy is highly dependent on competency of laboratory staff [2]. As a result, reinforcing strategies to promote good quality of microscopy-based malaria diagnosis has been gaining an increased interest in the global malaria agenda $[4,5]$.

In order to evaluate laboratory performance on malaria microscopy, WHO recognize the need to establish comprehensive external quality assessment (EQA) system [5]. However, little research on the evaluation of EQA systems in malaria microscopy has been conducted in Senegal. National Malaria Control Programs (NMCP) has defined a procedure to ensure good quality of malaria microscopy. This study aimed to evaluate the performances in malaria microscopy among laboratory technicians in Senegal.

\section{Methods}

\section{Description}

In Senegal, EQA procedures have been established by NMCP and regularly supervisory visits are conducted in all levels of malaria laboratory services. Also, NMCP organize periodic training towards lab technicians in conventional methods of malaria diagnosis. During the training sessions, participant laboratories are notified to store read slides in two separate provided boxes (Negative and Positive) for further quality control re-check. During the NMCP supervisory visits, senior microscopists assess the competence of laboratory technicians in performing thick and thin smear, reading slides, microscope maintenance and reagents management.

The collected results are analysed and reported to the NMCP, which conducts corrective actions for quality improvement.

\section{Sites and participants}

A cross-sectional study was conducted in three different strata of malaria distribution: (1) Saint Louis in the north (pre-elimination stage), (2) Dakar and Thies in the west centre (low prevalence) and (3) Kedougou in the south (high prevalence). Seventeen laboratories (seven hospitals and eleven health centres) participated (Fig. 1); Participants were lab technicians previously trained during training sessions conducted by NMCP.

\section{On-site evaluation}

A supervisory checklist was submitted to participants to assess the status of infrastructure, availability of comprehensive standard operating procedure (SOP), appropriate use of reagents and equipment, maintenance of microscope, laboratory safety, training related to malaria microscopy, data and supply management, internal quality control $(\mathrm{QC})$ procedure.

Also, supervisors examined that the slides are stored according to the appropriate SOPs and that the results are reported in a register with the following mentions: species identification (instead of mentioning "Positive results"), parasite stages (young trophozoites, mature trophozoites, schizonts and gametocytes), and assessment of parasite density.

\section{Slides rechecking}

Supervisors randomly selected 30 slides from each participant laboratory (15 samples of the smears from each negative and positive boxes where slides were stored). Seventeen laboratories were supervised. Two health centres were not able to store their read slides as required by NMCP so they were not included on the rechecking evaluation. Thus, a total of 450 slides ( 225 declared positives and 225 declared negatives) were collected from the 15 remaining laboratories. The selected smears were sent to the national reference laboratory (NRL) where they are blindly re-examined by two WHO level 1 malaria microscopists. Slides yielding discrepant results between two experts are resolved by a third expert microscopist.

\section{Proficiency testing}

Along with collecting stored slides, a set of eight reference slides was distributed to all involved participant microscopists: slide (1) Plasmodium falciparum moderate parasite density $(28,000 \mathrm{p} / \mu \mathrm{l})$ with Maurer dots (Fig. 2), slide (2) P. falciparum low parasite density $(192 \mathrm{p} / \mu \mathrm{l})$, slide (3) P. falciparum gametocyte and trophozoites with high parasite density and slides 4-8) five slides with no parasites.

The set of submitted slides consisted of reference slides from national malaria slide bank. The malaria slide bank is certified by $\mathrm{WHO}$ and is hosted in the Laboratory of 


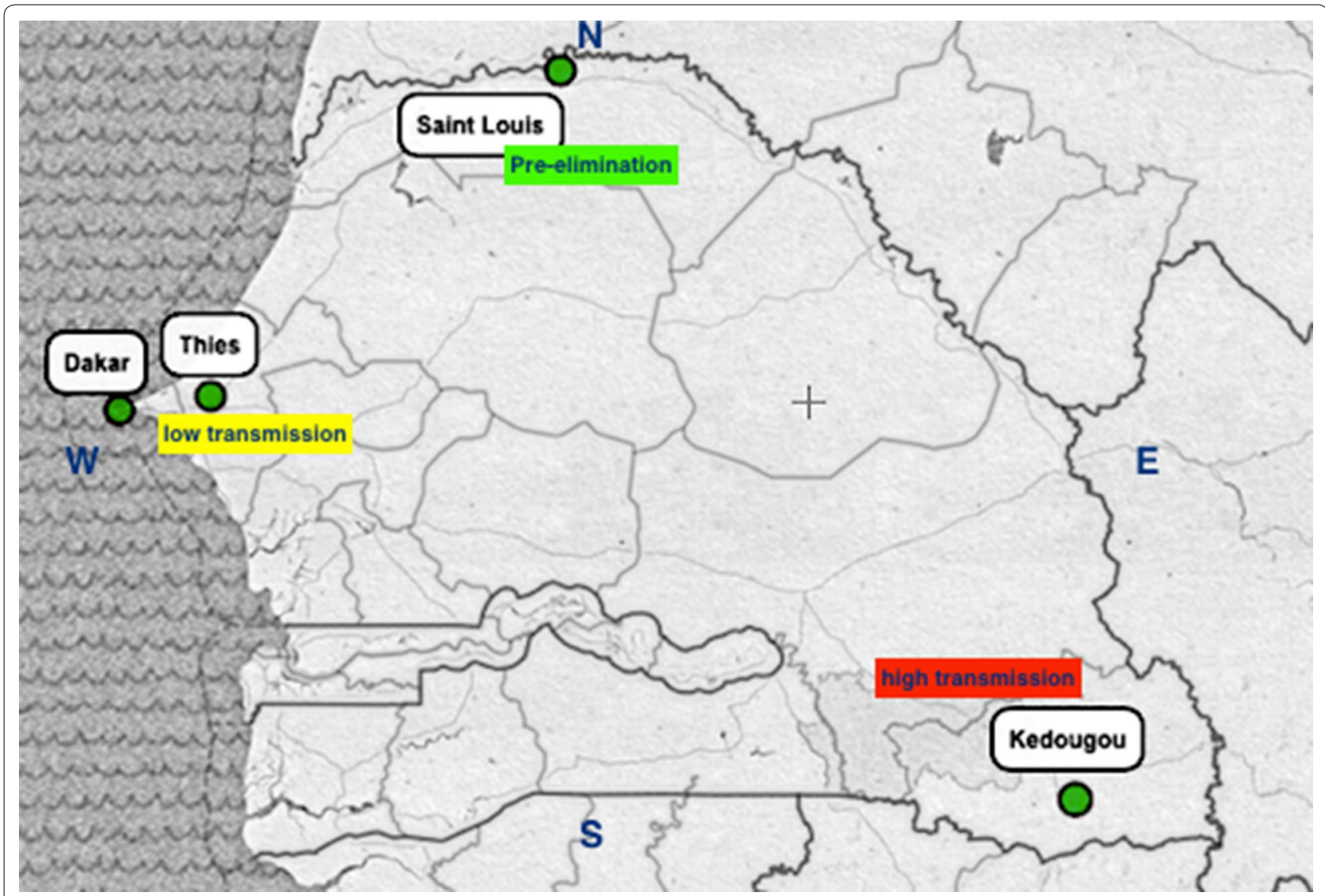

Fig. 1 Malaria epidemiological strata with sites where supervision was conducted; 7 hospital laboratories: Dakar (3), Thies (1) Saint Louis (2), Kedougou (1); 11 health centre laboratories: Dakar (2), Thies (2), Saint Louis (4), Kedougou (3)

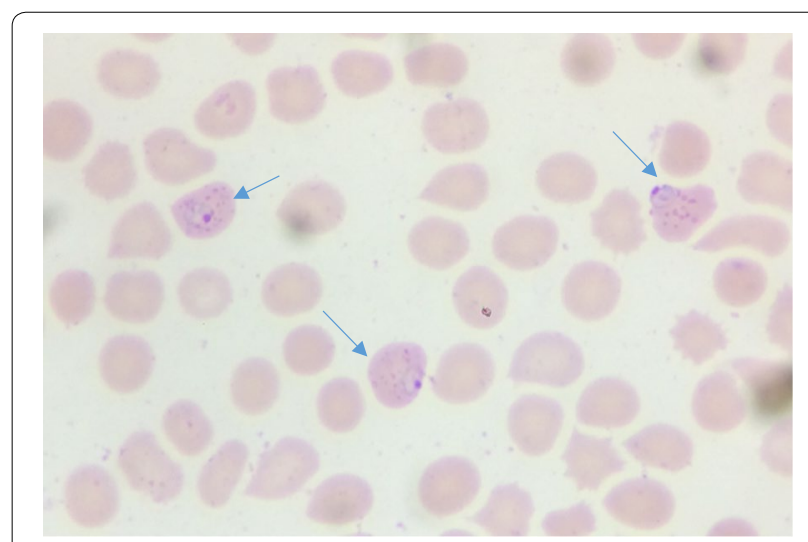

Fig. 2 Trophozoites of P. falciparum with specific Maurer dots inside the infected red blood cells, Giemsa stained thin smear $(\times 1000)$

Parasitology-Mycology of Aristide Le Dantec Hospital which is the national reference laboratory (NRL) for the Senegal NMCP. The NRL had six expert microscopists during past external competency assessment of malaria microscopy (ECAMM) courses.

\section{Data analysis}

The results of the EQA system were evaluated using the following indicators: major errors [high false-positive (HFP), high false-negative (HFN)], minor errors [low false-positive (LFP), low false-negative (LFN)] and misidentification; these indicators were defined depending on the modality of the evaluation:

\section{Slides rechecking}

LFP was defined as only one false positive is noted among the ten collected labelled negative by participants and HFP when there was more than one false positive. LFN was considered if the following slides were missed by participants among the ten labelled positive slides: parasite density less than $200 \mathrm{p} / \mu \mathrm{l}$, spirochetes, non-falciparum species. HFN was considered when slides with parasite density more than 200 was missed.

Misidentification refers to true positive slides that were detected positive by participants but species identification was not correct. 


\section{Proficiency testing}

LFP was defined when only one submitted reference negative slide is read positive by the participant and HFP when there were more than one false positive among the five negative slide. LFN was noted if only the slide with $P$. falciparum low parasite density $(192 \mathrm{p} / \mu \mathrm{l})$ was missed and HFN when slide with medium and high parasite density was missed by the participant.

Misidentification refers to reference positive slides that were detected positive by participants but species identification was not correct.

Sensitivity and specificity were calculated to assess the microscopy reading performance of the participants relative to the expert microscopy. Data were analysed using Excel (version 15.27). kappa statistic test was used to assess the magnitude of agreement between expert microscopists and participants. Exact Chi square analysis was used to determine the significance differences between sensitivities, specificities, negative predictive value (NPV) and positive predictive value (PPV). The p-values less than 0.05 were considered as statistical significance.

\section{Ethical consideration}

This study did not require informed consent from participants as no human subjects were involved. Ethical approval was obtained from Senegalese National Ethic Committee of Ministry of Health.

\section{Results}

\section{On-site evaluation}

Among the 17 participants, 14 laboratories have posted smear \& staining procedure and parasite counting method and 15 laboratories have arranged read slides in boxes accordingly to NMCP procedure. Two laboratories were using expired Giemsa reagent. Internal quality control (QC) was followed only by 1 laboratory.

All laboratories were using standardized laboratory register provided by NMCP; however, only 5 reported their results with complete information on the different sections of the registers; concerning the positive slide reporting results, 5 laboratories mentioned "Positive" without giving any details, 7 laboratories reported the specie "Plasmodium falciparum" without the differentiation stage. None laboratory mentioned the presence of gametocytes nor schizonts, although those stages were seen during re-examination process. Three participants did not report the parasite counting on their registers.

All reagents related to malaria microscopy were supplied directly through NMCP. All participant facilities were suitable for standards of infrastructure, safety and waste disposal practices. Microscope maintenance was respected by all laboratories.

\section{Proficiency testing}

Among the 17 participants, 11 laboratories scored 100\% as they correctly detected negative and positive reference slides. Three laboratories (one hospital and two health centres) misread negative slides as positive (LFN errors), four laboratories (one hospital and three health centres) misread low parasite density slide as negative (LFP errors); Two centres misread the $P$. falciparum slide with Maurer dots (Fig. 2) as $P$. ovale while one centre misread it as $P$. vivax; No major errors (HFP or HFN) were noted (Table 1).

\section{Cross-checking of slides}

Overall, 450 stored and collected slides from 15 participant laboratories were rechecked by WHO expert microscopists. Two health centres were excluded for this evaluation as they could not store their read slides as required by NMCP;

Eight laboratories achieved zero error. Only one major error was recorded as one health centre misread 2 negative slides as positive (HFP). Six laboratories recorded LFN results with the following positive slides misread as negative: spirochetes of Borrelia (missed by two hospital microscopists), $P$. ovale (missed by two health centres), and two slides with low parasite density of $P$. falciparum (missed by two health centres); the low parasite densities that were missed were 95 and $155 \mathrm{p} / \mu \mathrm{l}$. Three laboratories detected parasites on true positive slides but species identification was incorrect: two hospitals misidentified $P$. falciparum slides as $P$. malariae and one health centre participant misidentified $P$. ovale slide as $P$. vivax (Tables 2,3). When re-examining misidentified P. falciparum slides, expert microscopists observed "band-like forms" of trophozoites similar to that of P. malariae (Fig. 3); however, the absence of malarial pigments associated with high parasitaemia and the

\section{Table 1 Reported errors from participants on panel test- ing slides}

\begin{tabular}{|c|c|c|c|c|c|}
\hline Participants & Slide 1 & Slide 2 & Slide 3 & Slide 4-8 & Total errors \\
\hline Hospital 1 & Negative & & & P. falciparum & 2 \\
\hline Hospital 5 & & P. ovale & & & 1 \\
\hline $\mathrm{HC} 1$ & Negative & & & P. falciparum & 2 \\
\hline $\mathrm{HC} 5$ & & P. ovale & & & 1 \\
\hline $\mathrm{HC} 8$ & Negative & P. vivax & & P. falciparum & 3 \\
\hline HC 10 & Negative & & & & 1 \\
\hline Total errors & 4 & 3 & 0 & 3 & 10 \\
\hline
\end{tabular}

Slide 1: P. falciparum, $192 \mathrm{p} / \mu \mathrm{l}$

Slide 2: P. falciparum, 28,000 parasites with Maurer dots (see Fig. 1)

Slide 3: P. falciparum, with both trophozoites and gametocytes

Slides 4,5,6,7 and 8: No parasites 
Table 2 Errors reported during cross-checking of read slides

\begin{tabular}{|c|c|c|c|c|c|c|}
\hline \multirow[t]{2}{*}{ Participants laboratories } & \multicolumn{5}{|c|}{ Errors types } & \multirow[t]{2}{*}{ Total errors } \\
\hline & HFP & HFN & LFP & LFN $^{\mathrm{a}}$ & Misidentification $^{a}$ & \\
\hline Hospital 1 & 0 & 0 & 1 & 1 & 3 & 5 \\
\hline Hospital 2 & 0 & 0 & 0 & 1 & 0 & 1 \\
\hline Hospital 3 & 0 & 0 & 0 & 0 & 0 & \\
\hline Hospital 4 & 0 & 0 & 0 & 1 & 1 & 2 \\
\hline Hospital 5 & 0 & 0 & 0 & 0 & 0 & \\
\hline Hospital 6 & 0 & 0 & 0 & 0 & 0 & \\
\hline $\mathrm{HC} 1$ & 0 & 0 & 0 & 0 & 0 & \\
\hline $\mathrm{HC} 2$ & 2 & 0 & 0 & 0 & 0 & 2 \\
\hline $\mathrm{HC} 3$ & 0 & 0 & 0 & 0 & 0 & \\
\hline $\mathrm{HC} 4$ & 0 & 0 & 0 & 0 & 0 & \\
\hline HC 5 & 0 & 0 & 1 & 0 & 0 & 1 \\
\hline HC 6 & 0 & 0 & 0 & 0 & 1 & 1 \\
\hline $\mathrm{HC} 7$ & 0 & 0 & 0 & 0 & 0 & \\
\hline $\mathrm{HC} 8$ & 0 & 0 & 0 & 0 & 0 & \\
\hline $\mathrm{HC} 9$ & $N A$ & $N A$ & NA & $N A$ & $N A$ & \\
\hline HC 10 & $N A$ & $N A$ & $N A$ & $N A$ & $N A$ & \\
\hline HC 11 & 0 & 0 & 0 & 2 & 0 & 2 \\
\hline
\end{tabular}

$\mathrm{HC} 9$ and $\mathrm{HC} 10$ were not involved as they could not store the required number of slides

a See details on Table 3

Table 3 Results of missed slides and misidentified slides when controlled by expert microscopists

\begin{tabular}{lll}
\hline & Results of participants & Results of controllers \\
\hline Hospital 1 & Misidentification & \\
Hospital 4 & P. vivalariae & P. falciparum \\
& LFN & P. ovale \\
Hospital 1 & Negative & \\
Hospital 2 & Negative & Spirochetes \\
Hospital 4 & Negative & Spirochetes \\
HC 4 & Negative & P. falciparum, $95 \mathrm{p} / \mu \mathrm{l}$ \\
HC 11 & Negative & P. ovale \\
HC 11 & Negative & P. ovale \\
\hline
\end{tabular}

not-variegated aspect of the smear, were indicative of atypical $P$. falciparum.

The overall sensitivities and specificities for all participants against expert microscopists were 97.8 and $98.2 \%$ respectively; Sensitivities and specificities of hospital microscopists were 96.7 and $98.9 \%$ respectively while those of health centre microscopists were 98.5 and $97.8 \%$ respectively (Table 4). Overall, very good agreement was noted with kappa value of $0.96\left(\mathrm{CI}_{95 \%}\right.$ 93.4-98.6\%). No statistically significant differences of sensitivities and specificities were found between hospital and health centre microscopists $(\mathrm{p}=0.3993$ and $\mathrm{p}=0.9412$ respectively).

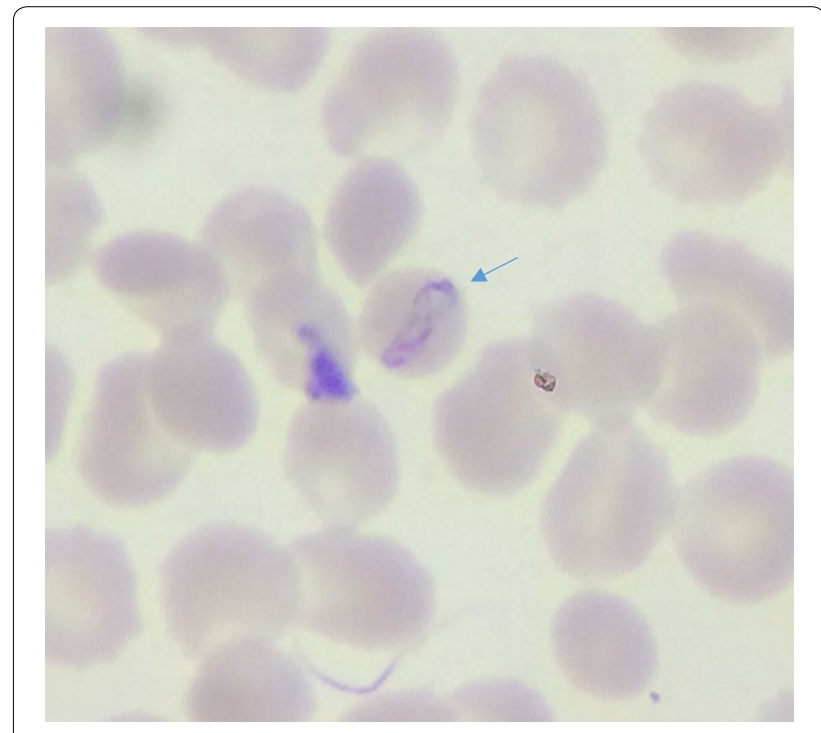

Fig. 3 P. falciparum trophozoite presenting an equatorial-like band in Giemsa stained thin smear $(\times 1000)$

\section{Discussion}

In Senegal, malaria transmission usually occurs during the rainy season (June-December) and the beginning of the dry season (January). This period corresponds to development of high density of vector populations. The prevalence of parasitaemia at the nation level have 
Table 4 Sensitivities, specificities, PPV and NPV of participant microscopists against expert microscopists

\begin{tabular}{llrrr}
\hline & & \multicolumn{2}{l}{ Controllers } & Total \\
\cline { 2 - 4 } & & Positive & Negative & \\
\hline Hospital technicians & Positive & 89 & 1 & 90 \\
& Negative & 3 & 87 & 90 \\
& Total & 92 & 88 & 180 \\
Sensitivity (\%) & 96.7 & & & \\
Specificity (\%) & 98.9 & & & \\
PPV (\%) & 98.9 & & & \\
NPV (\%) & 96.7 & & & \\
Health centers technicians & Positive & 132 & 3 & 135 \\
& Negative & 2 & 133 & 135 \\
& Total & 134 & 136 & 270 \\
Sensitivity (\%) & 98.5 & & & \\
Specificity (\%) & 97.8 & & & \\
PPV (\%) & 97.8 & & & \\
NPV (\%) & 98.5 & & & \\
\hline
\end{tabular}

decreased from $5.9 \%$ in 2008 to $2.8 \%$ in 2013 in relationship to policies carried out by NMCP. However, high disparities of malaria prevalence exist between endemic regions. Mainly, three different epidemiological profiles have been identified [6] in which three human infecting species, $P$. falciparum, P. malariae and $P$. ovale are known to circulate [7]. Low values of parasitaemia prevalence are concentrated in the northern Senegal, particularly in the region of Saint-Louis, Louga and Matam. Malaria risk increases in some areas of central Senegal and reaches the highest values in the southern (Kolda, Tambacounda and Kedougou) $[8,9]$. In the west centre, the parasite prevalence is about 1\% in Dakar and Thies [6]. The urban malaria burden is concentrated in the cities where the anopheles vector density is very low [8].

Malaria diagnosis is one of the key strategy for effective malaria control and elimination programs. Microscopy still remains the mainstay of malaria diagnosis in endemic countries [1]. Maintaining a quality-assured microscopy service is a major challenge [10]. The quality of microscopy-based malaria diagnosis has been questioned in several studies [11-14].

This study showed that malaria microscopy in Senegal had shown an overall low total errors during this supervisory visit, particularly when addressing $P$. falciparum detection. Senegal NMCP arranged regular training system, an effective supply management and regular periodic supervisions visits with on-site corrective actions; this most probably contributed to achieve this good performance.
In Senegal, for many years the NMCP targeted especially falciparum malaria to fight against malaria. Yet, it is well known that non-falciparum species are circulating in the country [15-17]. Moreover, WHO has recommended to integrate in the EQA other local pathogens that may be detected microscopically. In Senegal, Borrelia crocidurae, a bacterial spirochete, is a major cause of fever (thick-borne relapsing fever). The bacteria may be observed by experienced microscopists when examining routinely malaria thick and thin smears [18]. As a result, slides positive with spirochetes were included in the NRL malaria slide bank with the aim to train and evaluate malaria microscopists on this.

In this evaluation, the $P$. falciparum slide with Maurer dots was introduced on purpose to assess the skills of microscopists to recognize such granulations. In fact, most of the time in peripheral laboratories and even in hospital laboratories, Giemsa stain is diluted with tap water; in this condition, $P$. falciparum Maurer dots do not appear and lab technicians may be unfamiliar with those findings when slides are stained appropriately using buffered solution.

In endemic African countries it has been reported misidentifications of Plasmodium species, particularly for the non-falciparum species [11]. Difficulties to distinguish the non-falciparum species are common, especially for those with low prevalence [12]. Moreover, the NMCP tends to neglect non-falciparum malaria due to its less severe clinical picture compared to falciparum malaria [15].

The non-identification of the stages of $P$. falciparum was a real challenge. In fact, presence of gametocytes can be an indicator of therapeutic failure and even resistance to some antimalarial drugs [19]. Moreover, in low transmission areas where malaria elimination is planned, the proportion of individuals carrying gametocytes is critical to the maintain the transmission of the disease.

In Senegal, during the previous NMCP training sessions and proficiency testing, only P. falciparum slides were used to train malaria microscopists and to evaluate their performance. This supervisory report led the NMCP to consider revisions on the training format for the further sessions. For the first time, the training is supported by a validated reference slide set. Currently, the NRL through the malaria slide bank is able to provide reference slides for all non-falciparum species, falciparum low parasite densities, atypical trophozoites of $P$. falciparum and spirochetes of Borrelia.

Also, the NMCP initiated a program for microscopists to undergo WHO external competency assessment on malaria microscopy (ECAMM) courses; the first round allowed to identify a core group of microscopists (WHO level 1 and 2). Some of those leading microscopists have been selected to undergo training-of-facilitators courses. 
Thus, the next EQA should include more restringing requirements and this study could be considered as the baseline. Microscopists in the country will be assessed and certified in a national competence assessment (NCA) system. The protocol for these assessment and certification will include parasite density determination, nonfalciparum species identification, falciparum low parasite density detection, atypical trophozoites of $P$. falciparum recognition and spirochetes of Borrelia detection. It is expected, with this program, to increase trust among clinicians so they will prescribe appropriately antimalarial drugs [20]. A preceded retraining program is planned before evaluation. In fact, refresher training as well as adequate equipment and reagents, good working conditions, motivated and qualified personnel are known to improve microscopists skills [12, 14, 21].

In Senegal, according to NMCP procedure for crosschecking slides, the number of randomly selected slides is higher than that of WHO recommendations [22]. The advantage of a large number of taken slides is to increase the power of the study to detect slide reading errors; this is particularly relevant in regions striving to eliminate malaria, such as in north Senegal.

\section{Conclusion}

In Senegal, the performance quality of malaria microscopy is relatively satisfactory especially when addressing P. falciparum detection. However, non-falciparum species and blood bacterial such as Borrelia are likely to be missed when performing malaria microscopy. Another approaches are recommended to be adapted by future trainings and evaluations on microscopic laboratories.

\begin{abstract}
Abbreviations
ECAMM: external competency assessment of malaria microscopists; EQA external quality assessment; $\mathrm{HC}$ : health centre; HFN: high false-negative; HFP: high false-positive; LFN: low false-negative; LFP: low false-positive; NCA: national competence assessment; NMCP: national malaria control program; NPV: negative predictive value; NRL: national reference laboratory; PPV: positive predictive value; QC: quality control; RDT: rapid diagnostic test; SOP: standard operating procedures; WHO: World Health Organization.
\end{abstract}

\section{Authors' contributions}

$M A D, Y D, M C S$ and KD are WHO level 1 expert microscopists; they performed the microscopy re-examinations of taken slides. MAD drafted the manuscript; $M A D, B D, Y D, I D$ and YDN were involved in the fields during supervisory visits. ASB, ID and DN critically commented on the manuscript. All authors read and approved the final manuscript.

\section{Author details}

${ }^{1}$ Laboratory of Parasitology and Mycology, Cheikh Anta Diop University, Avenue Cheikh Anta Diop, BP 5005 Fann, Dakar, Senegal. ${ }^{2}$ National Malaria Control Program (NMCP), Rue Aimé Césaire, Fann Résidence, Dakar, Senegal.

\section{Acknowledgements}

We would like to thank Amy K. Bei for her help to English review this manuscript. We are thankful to the NMCP staff for their help to collect and transport slides.

\section{Competing interests}

The authors declare that they have no competing interests.

\section{Availability of data and materials}

The datasets supporting the conclusions of this article are included within the article.

\section{Consent for publication}

Consent for publication was not applicable as no human subjects were involved.

\section{Ethics approval and consent to participate}

The study received ethical clearance from the Ethic Committee of the Senegalese Ministry of Health. The study did not require informed consent from participants as no human subjects"'"were involved.

\section{Funding}

Not applicable.

\section{Publisher's Note}

Springer Nature remains neutral with regard to jurisdictional claims in published maps and institutional affiliations.

Received: 13 June 2017 Accepted: 8 March 2018

Published online: 15 March 2018

\section{References}

1. Maguire JD, Lederman ER, Barcus MJ, Wendy A, Meara PO, Jordon RG, Duong S, Muth S, Sismadi P, Bangs MJ, Prescott WR, Baird JK, Wongsrichanalai C. Production and validation of durable, high quality standardized malaria microscopy slides for teaching, testing and quality assurance during an era of declining diagnostic proficiency. Malar J. 2006;5:1-8.

2. Hawkes M, Kain KC. Advances in malaria diagnosis. Expert Rev Anti Infect Ther. 2007;5:485-95.

3. Trape JF, Diatta G, Arnathau C, Bitam I, Sarih M, Belghyti D, Bouattour A, Elguero E, Vial L, Mané Y, Baldé C, Pugnolle F, Chauvancy G, Mahé G, Granjon L, Duplantier JM, Durand P, Renaud F. The epidemiology and geographic distribution of relapsing fever borreliosis in West and North Africa, with a review of the Ornithodoros erraticus complex (Acari: Ixodida). PLOS ONE. 2013:8:1-19.

4. Ashraf S, Kao A, Hugo C, Christophel EM, Fatunmbi B, Luchavez J, Lilley K, Bell D. Developing standards for malaria microscopy: external competency assessment for malaria microscopists in the Asia-Pacific. Malar J. 2012;11:352.

5. WHO. Malaria microscopy quality assurance manual. 2nd ed. Geneva: WHO; 2015

6. PNLP. Cadre strategique national de lutte contre le paludisme AU Senegal 2014-2018. 2014

7. Lucchi NW, Gaye M, Diallo MA, Goldman IF, Ljolje D, Deme AB, Badiane A, Ndiaye YD, Barnwell JW, Udhayakumar V, Ndiaye D. Evaluation of the Illumigene Malaria LAMP: a robust molecular diagnostic tool for malaria parasites. Sci Rep. 2016;6(October):36808.

8. Giardina F, Gosoniu L, Konate L, Diouf MB, Perry R, Faye O, Vounatsou P. Estimating the burden of malaria in Senegal: Bayesian zero-inflated binomial geostatistical modeling of the MIS 2008 data. PLOS ONE. 2012;7:1-10.

9. Niang M, Thiam LG, Sow A, Loucoubar C, Bob NS, Diop F, Diouf B, Niass O, Mansourou A, Varela ML, Perraut R, Sall AA, Balde AT. A molecular survey of acute febrile illnesses reveals Plasmodium vivax infections in Kedougou, southeastern Senegal. Malar J. 2015;14:1-8.

10. Amexo M, Tolhurst R, Barnish G, Bates I. Malaria misdiagnosis: effects on the poor and vulnerable. Lancet. 2004;364:1896-8.

11. Mukadi $P$, Lejon $V$, Barbé $B$, Gillet $P$, Nyembo C. Performance of microscopy for the diagnosis of malaria and human African trypanosomiasis by diagnostic laboratories in the Democratic Republic of the Congo: Results of a Nation-Wide external quality assessment. PLOS ONE. 2016;11:e0146450. 
12. Obare P, Ogutu B, Adams M, Odera JS, Lilley K, Dosoo D, Adhiambo C, Owusu-agyei S, Binka F, Wanja E, Johnson J. Misclassification of Plasmodium infections by conventional microscopy and the impact of remedial training on the proficiency of laboratory technicians in species identification. Malar J. 2013;12:1-6.

13. Kahama-maro J, Acremont VD, Mtasiwa D, Genton B, Lengeler C. Low quality of routine microscopy for malaria at different levels of the health system in Dar es Salaam. 2011;10:332.

14. Biadglegne F, Belyhun Y, Ali J, Walle F, Gudeta N, Kassu A, Mulu A. Does the practice of blood film microscopy for detection and quantification of malaria parasites in northwest Ethiopia fit the standard? BMC Health Serv Res. 2014;14:529.

15. Diallo MA, Badiane AS, Diongue K, Deme A, Lucchi NW, Gaye M, Ndiaye T, Ndiaye M, Sene LK, Diop A, Gaye A, Ndiaye YD, Samb D, Yade MS, Ndir O, Udhayakumar V, Ndiaye D. Non-falciparum malaria in Dakar: a confirmed case of Plasmodium ovale wallikeri infection. Malar J. 2016:15:429.

16. Daniels RF, Deme AB, Gomis JF, Dieye B, Durfee K, Thwing Jl, Fall FB, Ba M, Ndiop M, Badiane AS, Ndiaye YD, Wirth DF, Volkman SK, Ndiaye D. Evidence of non-Plasmodium falciparum malaria infection in Kédougou, Sénégal. Malar J. 2017;16:9.
17. Badiane AS, Diongue K, Diallo S, Ndongo AA, Diedhiou CK, Deme AB, Ma D, Ndiaye M, Seck MC, Dieng T, Ndir O, Mboup S. Acute kidney injury associated with Plasmodium malariae infection. Malar J. 2014;13:1-5.

18. Diallo MA, Kane BS, Ndiaye M, Dieng M, Diongue K, Badiane AS, Seck MC, Ndiaye D. Plasmodium falciparum malaria co-infection with tick-borne relapsing fever in Dakar. Malar J. 2017;16:24.

19. White NJ. Antimalarial drug resistance. J Clin Invest. 2004;113:1084-92.

20. Joanny F, Löhr SJZ, Engleitner T, Lell B, Mordmüller B. Limit of blank and limit of detection of Plasmodium falciparum thick blood smear microscopy in a routine setting in Central Africa. Malar J. 2014;13:1-7.

21. Moura S, Fançony C, Mirante C, Neves M, Bernardino L, Fortes F, Sambo R, Brito M. Impact of a training course on the quality of malaria diagnosis by microscopy in Angola. Malar J. 2014;13:1-7.

22. Ekawati LL, Herdiana H, Sumiwi ME, Barussanah C, Ainun C, Sabri S, Maulana T, Rahmadyani R, Maneh C, Yani M, Valenti P, Elyazar IRF, Hawley WA. A comprehensive assessment of the malaria microscopy system of Aceh, Indonesia, in preparation for malaria elimination. Malar J. 2015;14:240

\section{Submit your next manuscript to BioMed Central and we will help you at every step:}

- We accept pre-submission inquiries

- Our selector tool helps you to find the most relevant journal

- We provide round the clock customer support

- Convenient online submission

- Thorough peer review

- Inclusion in PubMed and all major indexing services

- Maximum visibility for your research

Submit your manuscript at www.biomedcentral com/submit
() Biomed Central 